\title{
Identification of an Alternate Maxillary Apical Base Landmark from Pre-existing Substitution given by Different Authors
}

\author{
Dr Kunal Patel,' Dr Kartik Parikh,2 Dr Varun Pratap Singh,3 Dr Jay Soni \\ 1.4PG Student, ${ }^{2}$ Senior lecturer, Dept of Orthodontics, KM Shah Dental College, Vadodara, India \\ ${ }^{3}$ Assistant professor, Dept of Orthodontics, Nobel Medical College, Biratnagar, Nepal
}

Correspondence: Dr Kunal Patel; email: kunalortho@gmail.com

\section{ABSTRACT}

Introduction: It is often difficult to locate Point A in a lateral cephalogram due to operational errors. Faulty identification of Point A can lead to erroneous measurement and faulty diagnosis.

Objective: To identify nearest alternative maxillary apical base landmark for Point A substitutions given by different authors.

Materials \& Method: A cross sectional study was conducted on thirty good quality lateral cephalograms, which were appraised for skeletal Class I with the help of parameters angle ANB, WITS appraisal and Beta angle. Only those lateral cephalograms were selected where Point A was easily identified. Landmarks: Sella (S), Nasion (N), Point A and three substitution points Y, L, X were traced. Angles formed by SN with Point A (Angle SNA) and three substitution points (Angle SNY, SNX, SNL) were measured. Correlation of angle SNA with angles SNY, SNX and SNL were derived.

Result: A mean value of $82.8^{\circ} \pm 1.9^{\circ}, 83.1^{\circ} \pm 1.8^{\circ}, 78.3^{\circ} \pm 2.9^{\circ}$ and $78.7^{\circ} \pm 2.7^{\circ}$ for Angle's SNA, SNY, SNL and SNX respectively was observed. A statistically significant correlation was observed between angles SNA and SNY, SNL, SNX; and strong positive correlation was observed with angle SNY.

Conclusion: Point $Y$ is the most nearing maxillary apical base landmark to Point A. Hence maxillary apical base landmark can be substituted by Point $Y$ where identification of point $A$ is not obvious.

Key words: apical base, cephalometrics, Point A

\section{INTRODUCTION}

Cephalometric assessment of patients is an essential adjunct to achieve an accurate orthodontic diagnosis aiding for comprehensive orthodontic treatment planning. The extreme range or radiolucency between bone and soft tissues makes it impossible to locate consistently all landmarks on routine radiographs. Studies regarding the reliability of cephalometric landmarks have been differentiated by (1) differences between two films of the same subject, (2) observed differences in locating the points, and (3) variations in measuring the distance between two marked points.'

The factors influencing accurate identification were quoted as distinctness of structural detail, noise from adjacent structures due to superimposition of conflicting anatomic details, and conceptual judgment, a factor which is largely based on the past experience and radiological knowledge of the observer. ${ }^{2}$

In spite of improved techniques, occasionally certain landmarks are still difficult to locate, among them Point
A or Subspinale is one such landmark. Point A is a midline point whose relationship to the anterior teeth in a lateral head film may be influenced by head position. ${ }^{2}$ Almost all cephalometric analysis such as Steiner's, Down's, Wit's Appraisal, Mc Namara use point A or the NA plane as a reference point or plane. Because of the difficulty in locating point A; various authors like Van der Linden,' Jarabak and Fizzel, ${ }^{3}$ Jacobson and Jacobson ${ }^{4}$ have given different substitutions for Point $A$. The aim of this study was to identify the most nearing alternate maxillary apical base landmark from pre-existing cephalometric points given by different authors.

\section{MATERIALS AND METHOD}

The study was commenced after obtaining the approval from the Ethical Committee. A cross-sectional study was conducted on 30 (12 males; 18 females) pretreatment good quality lateral cephalograms from patients visiting to the Department of Orthodontics. Lateral cephalograms were selected such that the Point A could be accurately located. A purposive convenience sampling technique was used for the study. 
Patients above the age of 16 years were included in the sample. Patients with tooth agenesis or supernumeraries, developmental anomalies, traumatic injuries or fractured upper and lower incisors and molars, complex craniofacial deformities or syndromes, patients who have undergone orthodontic treatment were excluded from the study. The lateral cephalograms (Kodak $8000 \mathrm{C}$ Digital Panoramic and Cephalometric Systems) utilized in our study were of true size (1:1) and any faulty radiographs with image distortion were excluded. The exposure time ranged from 12.8 to 13.9 seconds with $\mathrm{kV}$ 69-71 and m/A 10-12. All cephalograms were manually traced by one investigator.

After categorization of the cephalograms; following landmarks were located and traced on acetate tracing paper: Sella (S), ${ }^{5}$ Nasion (N), ${ }^{5}$ Point A, ${ }^{6}$ Point $B, 5$ Point $Y,{ }^{4}$ Point $L^{1}$ and Point $X^{3}$ (Figure 1). Angles SNA, SNY, SNL and SNX were measured (Figure 2). Description of landmarks is given in Table 1.

Statistical tests were conducted using SPSS version 19.0. Mean and standard deviation for Angles SNA, SNY, SNL and SNX were calculated individually for males and females. t-test was applied to determine the statistical significance for all parameters i.e. Age, Angles SNA,
SNY, SNL and SNX respectively. Karl Pearson correlation coefficient was carried out to determine the correlation for Angle SNA with SNY, SNL and SNX.

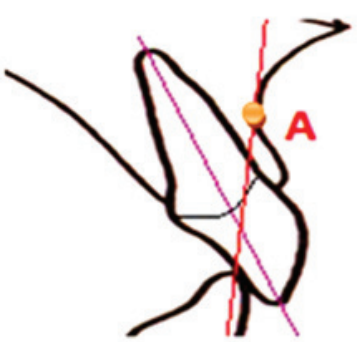

a

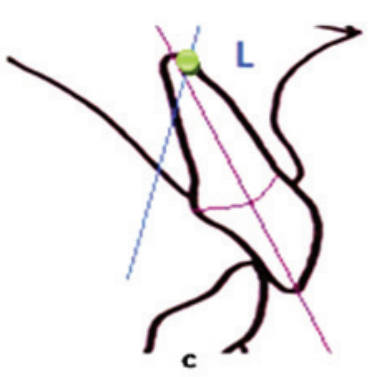

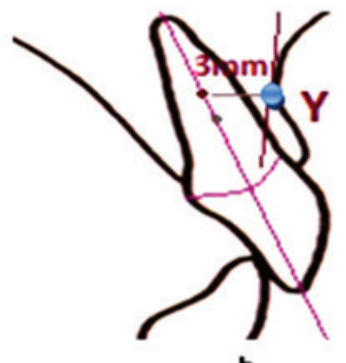

b

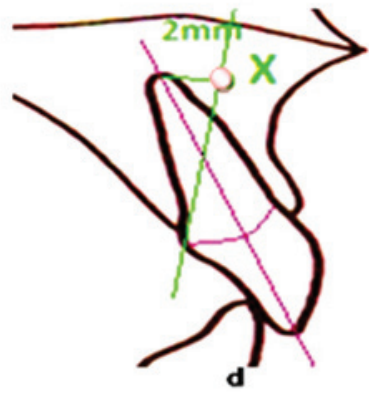

Figure 1: Various substitutions given by various authors
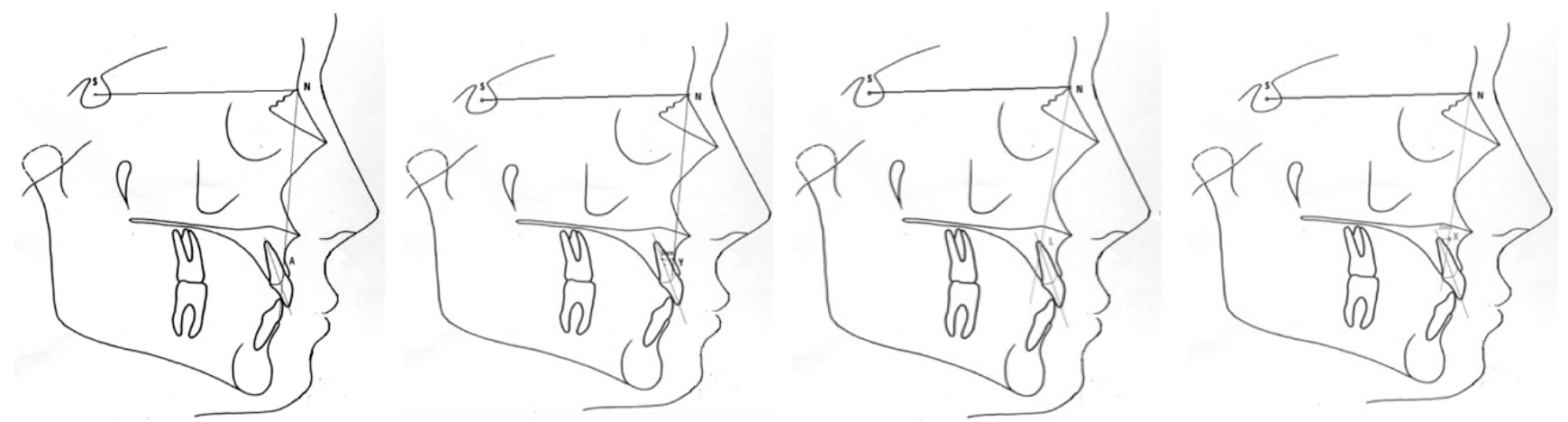

Figure 2: Various angles formed a: Angle SNA, b: Angle SNY, c: Angle SNL, d: Angle SNX

Table 1: Description of landmarks

\begin{tabular}{|l|l|}
\hline \multicolumn{1}{|c|}{ Landmark } & \multicolumn{1}{c|}{ Description } \\
\hline Sella $(\mathrm{S})$ & Geometric centre of the pituitary fossa \\
\hline Nasion $(\mathrm{N})$ & The most anterior point on the frontonasal suture in the midsagittal plane \\
\hline Point A or Subspinale & The deepest midline point on the premaxilla between anterior nasal spine and prosthion \\
\hline Point B or Supramentale & $\begin{array}{l}\text { The most posterior midline point in the concavity of the mandible between infradentale and } \\
\text { pogonion }\end{array}$ \\
\hline Point $Y$ & $\begin{array}{l}\text { Plotted } 3 \text { mm labial to a point between upper and lower two-thirds of the long axis of the root of } \\
\text { the maxillary central incisor }\end{array}$ \\
\hline Point $L$ & $\begin{array}{l}\text { Located on the anterior surface of the image of the labial lamella at the region of the apex of the } \\
\text { maxillary incisors }\end{array}$ \\
\hline Point $X$ & Located 2 mm ahead of the root apex of maxillary incisors \\
\hline
\end{tabular}




\section{RESULT}

In male samples; mean age was $22.1 \pm 4.3$ years. The mean values for angular measurements were obtained as: $82.8^{\circ} \pm$ $1.95^{\circ}$ for Angle SNA, 83.1 $\pm 1.8^{\circ}$ for Angle SNY, $78.3^{\circ} \pm 2.9^{\circ}$ for Angle SNL, and $78.7^{\circ} \pm 2.7^{\circ}$ for Angle SNX. In female samples; mean age was $19.8 \pm 3.2$ years. The mean values for angular measurements were obtained as: $80.9^{\circ} \pm 2.4^{\circ}$ for Angle SNA, $81.1^{\circ} \pm 2.5^{\circ}$ for Angle SNY, $77.3^{\circ} \pm 3.4^{\circ}$ for Angle SNL, and $77.9^{\circ} \pm 3.4^{\circ}$ for Angle SNX (Table 2).

Statistical analysis using t-test was carried out to determine the difference amongst all parameters: Age, SNA, SNY, SNL and SNX. Angles SNA $(\mathrm{P}<0.033)$ and SNY $(\mathrm{P}<0.023)$ revealed statistical significance (Table 3).

The correlation of Age, SNA, SNY, SNL and SNX was calculated. Age had non-significant correlation with all the parameters utilized in the study. SNA had strong statistically significant correlation with SNY, SNX, SNL (Table 4).

In males, age had non-significant correlation with all the parameters utilized in the study. SNA revealed a strong correlation with SNY only (Table 5). In females, age had nonsignificant correlation with all the parameters utilized in the study. SNA revealed a strong correlation with SNY, SNL and SNX (Table 6).

Table 2: Descriptive statistics for parameters SNA, SNY, SNL, SNX and Age among male and female subjects

\begin{tabular}{|l|l|l|l|l|c|}
\hline & \multicolumn{1}{|c|}{ Sex } & N & Mean & SD & SEM \\
\hline \multirow{3}{*}{ SNA } & Male & 12 & 82.87 & 1.95 & 0.5645 \\
\cline { 2 - 6 } & Female & 18 & 80.97 & 2.45 & 0.5794 \\
\hline \multirow{3}{*}{ SNY } & Male & 12 & 83.08 & 1.85 & 0.5359 \\
\cline { 2 - 6 } & Female & 18 & 81.02 & 2.54 & 0.5988 \\
\hline \multirow{2}{*}{ SNL } & Male & 12 & 78.29 & 2.87 & 0.8313 \\
\cline { 2 - 6 } & Female & 18 & 77.33 & 3.36 & 0.7931 \\
\hline \multirow{3}{*}{ SNX } & Male & 12 & 78.75 & 2.75 & 0.7965 \\
\cline { 2 - 6 } & Female & 18 & 77.94 & 3.47 & 0.8193 \\
\hline \multirow{2}{*}{ Age } & Male & 12 & 22.16 & 3.32 & 1.2482 \\
\cline { 2 - 6 } & Female & 18 & 19.77 & 3.19 & 0.7521 \\
\hline
\end{tabular}

Table 3: t-test of significance for parameters SNA, SNY, SNL, SNX and Age

\begin{tabular}{|l|c|c|c|}
\hline & $\boldsymbol{t}$-Value & DF & $\boldsymbol{p}$-Value \\
\hline SNA & 2.245 & 28 & 0.033 \\
\hline SNY & 2.402 & 28 & 0.023 \\
\hline SNL & 0.808 & 28 & 0.426 \\
\hline SNX & 0.673 & 28 & 0.507 \\
\hline Age & 1.743 & 28 & 0.092 \\
\hline
\end{tabular}

Table 4: Determination of correlation coefficient for parameters SNA, SNY, SNL, SNX and Age

\begin{tabular}{|l|l|c|c|c|c|c|}
\hline \multicolumn{2}{|c|}{ Age } & Age & SNA & SNY & SNL & SNX \\
\cline { 2 - 8 } & Pearson Correlation & 1 & 0.057 & 0.001 & -0.061 & -0.119 \\
\hline \multirow{2}{*}{ SNA } & P-value & - & 0.763 & 0.995 & 0.749 & 0.530 \\
\cline { 2 - 8 } & Pearson Correlation & 0.057 & 1 & $0.963^{* *}$ & $0.706^{* *}$ & $0.725^{* *}$ \\
\hline
\end{tabular}

$\mathrm{N}=30,{ }^{* *}$ Correlation is significant at 0.01 level (2-tailed) 
Table 5: Determination of correlation coefficient for parameters SNA, SNY, SNL, SNX and Age for females

\begin{tabular}{|l|l|c|c|c|c|c|}
\hline \multicolumn{2}{|c|}{} & Age & SNA & SNY & SNL & SNX \\
\hline \multirow{2}{*}{ Age } & Pearson Correlation & 1 & -0.035 & -0.133 & 0.081 & -0.020 \\
\cline { 2 - 8 } & P-value & - & 0.892 & 0.598 & 0.749 & 0.938 \\
\hline \multirow{2}{*}{ SNA } & Pearson Correlation & -0.035 & 1 & $0.968^{* *}$ & $0.883^{* *}$ & $0.898^{* *}$ \\
\cline { 2 - 8 } & p-value & 0.892 & - & 0.000 & 0.000 & 0.000 \\
\hline
\end{tabular}

$N=18 ; * *$ Correlation is significant at 0.01 level (2 tailed)

Table 6: Determination of correlation coefficient for parameters SNA, SNY, SNL, SNX and Age for males

\begin{tabular}{|l|l|c|c|c|c|c|}
\hline \multicolumn{2}{|c|}{ Age } & Age & SNA & SNY & SNL & SNX \\
\cline { 2 - 8 } & Pearson Correlation & 1 & -0.137 & -0.189 & -0.384 & -0.396 \\
\hline \multirow{2}{*}{ SNA } & Pealue & - & 0.671 & 0.557 & 0.218 & 0.202 \\
\cline { 2 - 8 } & P-value & -0.137 & 1 & $0.930^{* *}$ & 0.322 & 0.352 \\
\hline
\end{tabular}

$\mathrm{N}=12 ;{ }^{* *}$ Correlation is significant at 0.01 level (2 tailed)

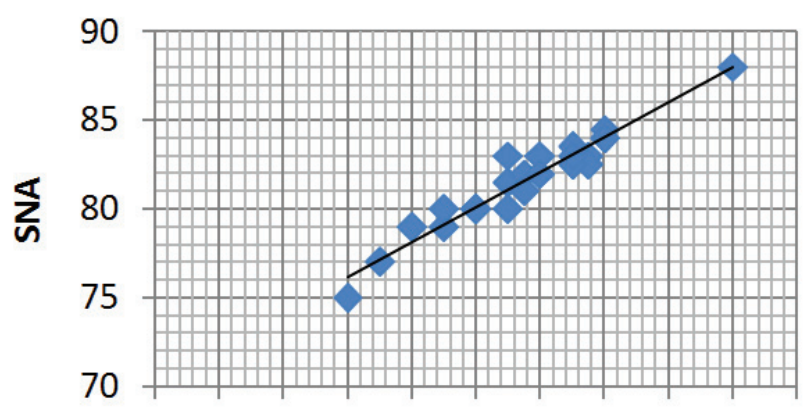

$\begin{array}{lllllllllll}70 & 72 & 74 & 76 & 78 & 80 & 82 & 84 & 86 & 88 & 90\end{array}$

SNY

Graph 1: Scatter diagram showing correlation between angles SNA and SNY

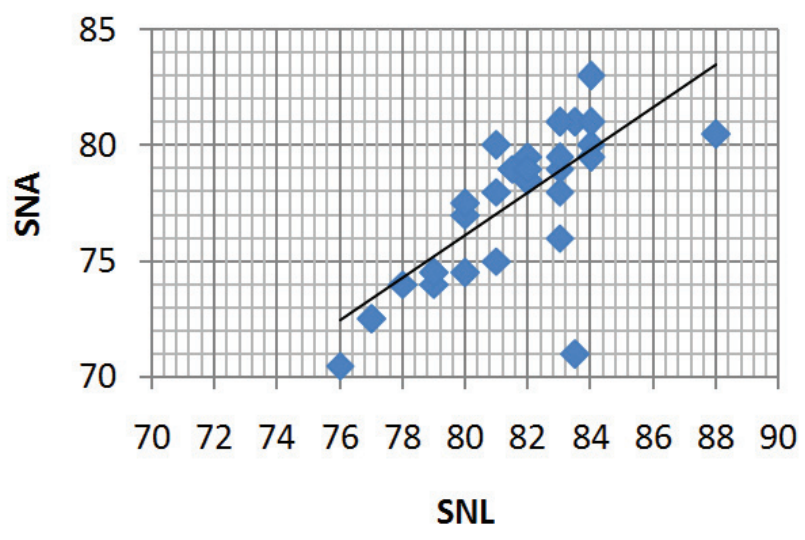

Graph 2: Scatter diagram showing correlation between angles SNA and SNL

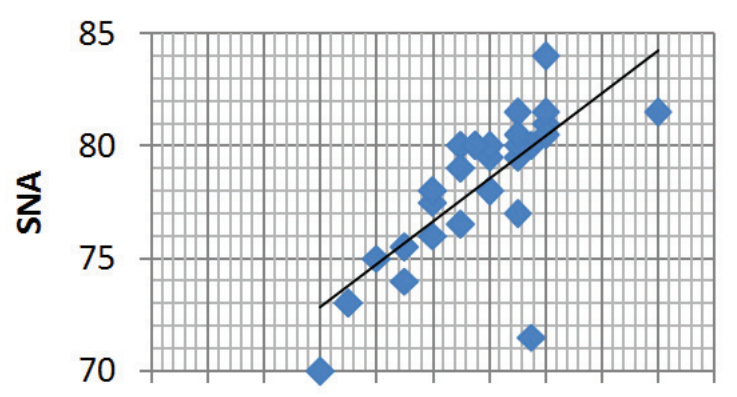

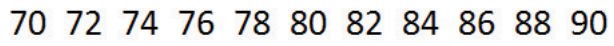

SNX

Graph 3: Scatter diagram showing correlation between angles SNA and SNX 


\section{DISCUSSION}

Apical base of maxilla and mandible help in determining the spatial relation of both maxilla and mandible to the crania base. It also determines the limit of placement of incisors in the antero-posterior position. ${ }^{7}$ Numerous controversies exist in landmarks which are difficult to identify. Among those, Point $A$ is the most common point which encounters difficulty in identification.

With the advent of digital cephalometry, landmarks were made easily visualized and identified. The cephalometric landmark, Point A was investigated with regard to definition, location and usefulness in cephalometric analysis. Point A or Subspinale represents the maxillary apical base; the projection of cheeks frequently obscures this landmark in lateral cephalogram. ${ }^{4}$ Due to shortcomings of Point A, various substitute landmarks have been sought by different authors by keeping the root apex of maxillary central incisor as a stable landmark. Van der Linden suggested the use of point $L$, which is located on the anterior surface of the image of the labial lamella at the region of the apex of maxillary incisors. Jarabak and Fizzel identified Point $X$, which is $2 \mathrm{~mm}$ ahead of the root apex as a redefinition of point A. ${ }^{3}$ Jacobson \& Jacobson suggested another Point $Y$, plotted $3 \mathrm{~mm}$ labial to a point between upper and lower two-thirds of the long axis of the root of maxillary central incisor. ${ }^{4}$

When the mean values of angles SNA, SNL, SNX and SNY were compared; the mean value of SNY was more in comparison to SNA, SNL and SNX. The mean values of SNL and SNX was less in comparison to SNA. Probable cause for such observation could be attributed to the variation in the definition of those cephalometric landmarks.

When t-test was carried out to determine the significance amongst all parameters individually; Angles SNA and SNY revealed statistical significance. This suggested that angles SNA and SNY were more specific for all the samples included in the study.

Among all; SNA and SNY, and SNX and SNL revealed strong statistical correlation between each other. In males there was high correlation of angle SNA with angle SNY. In females, angle SNA correlated with Angle SNY, SNL and SNX. We observed that all angles were less in females compared to the males. However a further study needs be conducted to ascertain the probable cause for such an observation.

In almost all cases, the angles SNA and SNY were equal and showed high significant correlation. Al-Abdwani et $a^{8}$ stated that the effects of incisal inclination changes due to orthodontic treatment are of no clinical relevance to the position of Point A and B, even though they may be statistically significant. However Kazem et $a^{19}$ reported that the position of Point $A$ is affected by local bone remodeling associated with proclination of the upper incisor in Class II Division 2 malocclusion, but this minor change does not significantly affect SNA angle. According to Jacobson ${ }^{4}$ a point closer to the center of the root of a tooth is less vulnerable to displacement than, say, a point close to the root apex during crown tipping procedures. Point $Y$ represents closer to center of the tooth root hence point $Y$ can be used more precisely as a substitute for point $A$. Whereas Point $L$ and Point $X$ are located in relation to the root apex, which can change if the tooth is proclined or retroclined.

\section{CONCLUSION}

The situation of cephalometric Point $A$ is complex, and its location depends on a number of variables. Thus from the present study the following conclusions can be drawn:

1. Age has no significance on identification of maxillary apical base.

2. Point A shows statistically strong correlation with Point $Y$.

3. In males, Point $A$ and Point $Y$ are strongly correlated.

4. In females, Point A showed significant correlation with Point $Y, L$ and $X$.

Thus, point $Y$ given by Jacobson ${ }^{4}$ is the most suitable landmark which can be substituted for Point A amongst all other points suggested by different authors. In instances where Point $A$ is not clearly discernible an alternative Point $Y$ which is located $3 \mathrm{~mm}$ labial to a point between the upper third and lower two third of the long axis of the root of the maxillary central incisor.

\section{REFERENCES}

1. Van der Linden FPGM. A study of roentgenographic bony landmarks. Am J Orthod 1971; 59:111-125.

2. Baumrind, S, Frantz RC. The reliability of head film measurements. 1. Landmark identification. Am J Orthod 1971; 60:1 11

3. Jarabak J.R, Fizzel. Technique and treatment with the light wire appliance. St. Louis, C.V. Mosby Company. 1963 ; pp. 146.

4. Jacobson RL, Jacobson A. Point A revisited. Am J Orthod. 1980; 77(1).

5. Jacobson A. Radiographic cephalometry. 2nd Ed. Quintessence Publishing, 2006.

6. Downs WB. Variations in facial relationship: Their significance in treatment and prognosis. Am J Orthod 1948; 34: 812-840.

7. Kalafa JA, Kronman JH. A Critical Evaluation of Cephalometric "A" Point and Proposal of a More Significant Landmark. Angle Orthod 1968: 38(3):225-230.

8. Al-Abdwani R. Change of incisor inclination effects on Points A and B. Angle Orthod 2009; 79:462-467.

9. Kazem S. Al-Nimri. Maxillary incisor proclination effect on the position of Point A in Class II Division 2 malocclusion. Angle Orthod 2009: 79:880-884. 